\title{
CONVERGENCE ANALYSIS OF SMOOTHING METHODS FOR OPTIMAL CONTROL OF STATIONARY VARIATIONAL INEQUALITIES WITH CONTROL CONSTRAINTS *
}

\author{
Anton Schiela ${ }^{1}$ AND DANIEl WAChSMUth ${ }^{2}$
}

\begin{abstract}
In the article an optimal control problem subject to a stationary variational inequality is investigated. The optimal control problem is complemented with pointwise control constraints. The convergence of a smoothing scheme is analyzed. There, the variational inequality is replaced by a semilinear elliptic equation. It is shown that solutions of the regularized optimal control problem converge to solutions of the original one. Passing to the limit in the optimality system of the regularized problem allows to prove C-stationarity of local solutions of the original problem. Moreover, convergence rates with respect to the regularization parameter for the error in the control are obtained, which turn out to be sharp. These rates coincide with rates obtained by numerical experiments, which are included in the paper.
\end{abstract}

Mathematics Subject Classification. 49K20, 65K15, 49M20, 90C33.

Received June 20, 2011. Revised September 11, 2012.

Published online March 4, 2013.

\section{INTRODUCTION}

In this article we analyze a regularization algorithm to solve the following non-smooth optimal control problem: Minimize the function $J$ given by

$$
J(y, u)=g(y)+j(u)
$$

over all $(y, u) \in K \times L^{2}(\Omega)$ subject to the elliptic variational inequality

$$
\langle A y, v-y\rangle \geq(u, v-y) \quad \forall v \in K
$$

and the control constraints

$$
u \in U_{a d}:=\left\{u \in L^{2}(\Omega): u_{a} \leq u \leq u_{b} \quad \text { a.e. on } \quad \Omega\right\} .
$$

Keywords and phrases. Variational inequalities, optimal control, control constraints, regularization, C-stationarity, pathfollowing.

* Supported by the DFG Research Center Matheon "Mathematics for key technologies".

1 Konrad-Zuse-Zentrum für Informationstechnik Berlin(ZIB), Takustraße 7, 14195 Berlin-Dahlem, Germany. schiela@zib.de; http://www.zib.de/schiela,

2 Johann Radon Institute for Computational and Applied Mathematics (RICAM), Austrian Academy of Sciences, Altenbergerstraße 69, A-4040 Linz, Austria. daniel.wachsmuth@oeaw.ac.at; http://www.ricam.oeaw.ac.at/people/page/wachsmuth 
The feasible set $K$ is given by

$$
K=\left\{v \in H_{0}^{1}(\Omega): v \leq \psi\right\} .
$$

Here, $\Omega \subset \mathbb{R}^{n}, n=2,3$, is a bounded Lipschitz domain. The pairings $\langle\cdot, \cdot\rangle$ and $(\cdot, \cdot)$ are short-hand notations for the duality pairing in $H^{-1}(\Omega) \times H_{0}^{1}(\Omega)$ and the scalar product in $L^{2}(\Omega)$, respectively. The operator $A$ is an elliptic second-order differential operator. The function $\psi \in H^{1}(\Omega) \cap L^{\infty}(\Omega)$ represents the obstacle. The assumptions on the various ingredients of the optimization problem $(\mathrm{P})$ will be made precise below.

The optimization problem $(\mathrm{P})$ is a non-smooth and non-convex optimization problem, which makes it challenging to prove sharp first-order necessary optimality conditions. In addition, it is difficult to develop fast solution algorithms to solve $(\mathrm{P})$.

In the present paper, we address both of these questions. We investigate a smoothing scheme for $(\mathrm{P})$ that is used to compute numerical approximations. We study its convergence and prove sharp convergence rates, which improve former results of [16]. In addition, we prove that the limit of primal and dual quantities satisfies a certain first-order necessary optimality system, which extends available results $[8,11,13,17,20]$.

\section{First-order necessary conditions}

Let us first explain the difficulties that appear when analyzing the optimal control problem (P). Under suitable assumption classical results guarantee existence of unique solutions of the variational inequality (1.1). Introducing a multiplier $\lambda$, the variational inequality can be written equivalently as

$$
A y+\lambda=u, \quad y \leq \psi, \lambda \geq 0,\langle\lambda, y-\psi\rangle=0,
$$

where $\lambda \in H^{-1}$, and $\lambda \geq 0$ is short for $\langle\lambda, v\rangle \geq 0$ for all $v \in H_{0}^{1}(\Omega)$, with $v \geq 0$. With the reformulation (1.4), the problem $(\mathrm{P})$ is an optimization problem subject to a complementary condition constraint. Such problems are known to violate standard constraint qualifications of nonlinear programming, and thus it is challenging to derive necessary optimality conditions.

As local optimal solutions are characterized by necessary optimality conditions, there is much interest in finding the strongest possible optimality condition. In order to distinguish different stationarity concepts, we follow the nomenclature of [21].

Definition 1.1. The point $\left(y^{*}, u^{*}, \lambda^{*}\right) \in K \times U_{a d} \times L^{2}(\Omega)$ is called C-stationary for (P) if it satisfies (1.4) and if there exist $\left(p^{*}, \mu^{*}\right) \in\left(H_{0}^{1}(\Omega) \cap L^{\infty}(\Omega)\right) \times\left(H^{-1}(\Omega) \cap C(\bar{\Omega})^{*}\right)$ such that the following system is fulfilled:

$$
\begin{gathered}
A^{*} p^{*}+\mu^{*}=g^{\prime}\left(y^{*}\right), \\
\left(j^{\prime}\left(u^{*}\right)+p^{*}, u-u^{*}\right) \geq 0 \quad \forall u \in U_{a d}, \\
p^{*}=0 \quad \text { a.e. on }\left\{x \in \Omega: \lambda^{*}>0\right\}, \\
\left\langle\mu^{*}, \phi\right\rangle_{C(\bar{\Omega})^{*}, C(\bar{\Omega})}=0 \forall \phi \in C(\bar{\Omega}): \phi=0 \quad \text { a.e. on } \quad\left\{x \in \Omega: y^{*}=\psi\right\} \\
\left\langle\mu^{*}, \phi p^{*}\right\rangle_{C(\bar{\Omega})^{*}, C(\bar{\Omega})} \geq 0 \forall \phi \in C(\bar{\Omega}): \phi \geq 0 .
\end{gathered}
$$

The point $\left(y^{*}, u^{*}, \lambda^{*}\right)$ is called strongly stationary if in addition

$$
\begin{gathered}
p^{*} \leq 0 \quad \text { a.e. on } \quad B, \\
\left\langle\mu^{*}, \phi\right\rangle \leq 0 \quad \text { for all } \quad \phi \in H_{0}^{1}(\Omega):\left(\lambda^{*}, \phi\right)=0, \phi \geq 0 \quad \text { a.e. on } \quad B,
\end{gathered}
$$

is satisfied, where $B$ is the biactive set

$$
B:=\left\{x \in \Omega: y^{*}=\psi, \lambda^{*}=0\right\} .
$$


Here, the notation 'strong stationarity' refers to the fact, that it is the strongest optimality condition, which can be expected to hold at a solution of $(\mathrm{P})$. Strong stationarity and C-stationarity differ in the conditions on the sign of the dual quantities $p^{*}$ and $\mu^{*}$ on the bi-active set.

As optimal control problems constrained by a variational inequality were studied over the last decades, there is some work available on necessary optimality conditions. Here, we refer to Barbu [1], Bergounioux [2], Hintermüller and Kopacka [8], Hintermüller, Mordukhovich, and Surowiec [10], Hintermüller and Surowiec [11], Ito and Kunisch [13], Mignot [18], Mignot and Puel [19], Outrata, Jarušek, and Stará [20]. Sufficient optimality conditions were studied by Kunisch and Wachsmuth [17].

Strong stationarity is obtained by Mignot [18] and Mignot and Puel [19] for optimal control problems without control constraints, that is for the special case $U_{a d}=L^{2}(\Omega)$. For more general situations, it is difficult to prove the relations (1.6a) and (1.6b) on the biactive set. In [13] C-stationarity is proven for the case without control constraints, with (1.5e) replaced by the weaker condition $\left\langle\mu^{*}, p^{*}\right\rangle \geq 0$. For the two-dimensional case with control constraints C-stationarity is proven in [20] again with (1.5e) replaced by $\left\langle\mu^{*}, p^{*}\right\rangle \geq 0$. The research report [8] proves an even weaker variant for limit points of sequences obtained by a regularization method. Using limiting variational calculus [10] prove limiting stationarity conditions, which involve the alternative $\mu^{*} \geq 0$ or $\mu^{*} \leq 0$ on the active set. However, from none of these works, C-stationarity in the sense of Definition 1.1 can be derived directly. The present article thus complements previous works, as we prove C-stationarity in Theorem 3.9 below.

Let us argue that strong stationarity is not a necessary optimality condition for problem $(\mathrm{P})$, the example is taken from [23]. To this end, choose constant control bounds $u_{a}=0$ and $u_{b}=1$. With the choice $A=-\Delta$ and $\psi=0$, it follows by the maximum principle that the solution $y(u)$ of (1.1) is zero for all $u \in U_{a d}$. Let $j(u):=\frac{1}{2}\|u\|_{L^{2}(\Omega)}^{2}, g(y):=\frac{1}{2}\left\|y-y_{d}\right\|_{L^{2}(\Omega)}^{2}$ with $y_{d}=-1$. Then $y^{*}=u^{*}=\lambda^{*}=0$ is a solution of the optimal control problem. Due to the result of Theorem 3.9, C-stationarity is necessary for local optimality thus we obtain the existence of $\left(p^{*}, \mu^{*}\right)$ satisfying (1.5a)-(1.5e). The inequality directly yields $p^{*} \geq 0$. Let us assume that strong stationarity holds. Then $p^{*} \leq 0$ and $\mu^{*} \leq 0$ follows by (1.6a)-(1.6b), since $B=\Omega$, which immediately gives $p^{*}=0$. From (1.5a) we obtain $-\Delta p^{*}+\mu^{*}=y^{*}-y_{d}$, which implies $\mu^{*}=-y_{d}=+1$, which is a contradiction. This proves that in general strong stationarity is not a necessary optimality condition for $(\mathrm{P})$.

\section{Smoothing scheme}

Due to the appearance of multipliers, which are only measures, and as a consequence of the complementarity conditions (1.4) and (1.5c)-(1.6b), stationarity systems are not well-suited for numerical realization. Algorithms to solve $(\mathrm{P})$ are then based on a suitable smoothing of the underlying constraints. Hintermüller and Kopacka [8] used a relaxation scheme, where $\langle\lambda, y-\psi\rangle=0$ is replaced by $\langle\lambda, y-\psi\rangle \leq \alpha$, coupled with a Moreau-Yosida regularization of the resulting state-constrained problem. Another approach is based on the following equivalent reformulation of the complementarity constraint in (1.4):

$$
\lambda=\max (0, \lambda+c(y-\psi)) \quad \forall c>0 .
$$

The max-function is then replaced by a suitable smooth approximation. This approach is followed in Hintermüller and Kopacka [9], Ito and Kunisch [13], and Kunisch and Wachsmuth [16]. Such smoothing techniques were also applied in the past to derive existence and regularity of solutions of variational inequalities, see e.g. Friedman [7] and Kinderlehrer and Stampacchia [15].

In this paper, we will use this smoothing approach and apply it to the original problem. That is, we replace the non-smooth condition $\lambda=\max (0, \lambda+c(y-\psi))$ by

$$
\lambda_{c}=\max _{c}(0, \bar{\lambda}+c(y-\psi)) .
$$

Here, $c$ is the regularization and smoothing parameter. Moreover, $\bar{\lambda} \in L^{\infty}(\Omega)$ is a given non-negative function, and $\max _{c}$ is a $C^{2}$-approximation of $x \rightarrow \max (0, x)$, which is made precise below. For properly chosen $\bar{\lambda}$ the solutions $y_{c}$ of the regularized equation are feasible, i.e. $y_{c} \leq \psi$, see $[13,16,17]$. 
The resulting family of regularized problems is then given by

$$
\left\{\begin{array}{c}
\min J(y, u) \\
\text { over }(y, u) \in H_{0}^{1}(\Omega) \times U_{a d} \text { subject to } \\
A y+\max _{c}(0, \bar{\lambda}+c(y-\psi))=u .
\end{array}\right.
$$

An important observation is that this problem does not incorporate any inequality constraints on $y_{c}, \lambda_{c}$, and $\lambda_{c}\left(y_{c}-\psi\right)$.

If $g$ and $j$ are $C^{1}$-regular, then the first order optimality system for $\left(\mathrm{P}_{c}\right)$ is given by

$$
\begin{gathered}
A y_{c}+\max _{c}\left(\bar{\lambda}+c\left(y_{c}-\psi\right)\right)=u_{c}, \\
A^{*} p_{c}+c \max _{c}^{\prime}\left(\bar{\lambda}+c\left(y_{c}-\psi\right)\right) p_{c}=g^{\prime}\left(y_{c}\right), \\
\left(j^{\prime}\left(u_{c}\right)+p_{c}, u-u_{c}\right) \geq 0 \quad \forall u \in U_{a d} .
\end{gathered}
$$

In $[14,17]$ existence of solutions $\left(y_{c}, u_{c}\right) \in H_{0}^{1}(\Omega) \times L^{2}(\Omega) \times H_{0}^{1}(\Omega)$ to $(1.8)$ for the case $U_{a d}=L^{2}(\Omega)$ was established and subsequential convergence for $c \rightarrow \infty$ to a solution $\left(y^{*}, u^{*}\right)$ of the unregularized problem was argued. For the same subsequence we have

$$
\lambda_{c} \rightarrow \lambda^{*} \text { and } \mu_{c} \rightarrow \mu^{*} \text { in } H^{-1}(\Omega), p_{c} \rightarrow p^{*} \text { in } H_{0}^{1}(\Omega),
$$

where $\rightarrow$ and $\rightarrow$ denote strong and weak convergence respectively, and the multiplier approximations $\lambda_{c}$ and $\mu_{c}$ are defined by

$$
\lambda_{c}=\max _{c}\left(0, \bar{\lambda}+c\left(y_{c}-\psi\right)\right), \quad \mu_{c}=c \max _{c}^{\prime}\left(\bar{\lambda}+c\left(y_{c}-\psi\right)\right) p_{c} .
$$

Moreover, one can show that each strict local minimum of $(\mathrm{P})$ is the (weak) limit of a sequence of solutions of the regularized problem [17]. Hence, one can use these sequences to derive optimality conditions for the original problem. In this paper, we will extend these results to the control constrained case and prove C-stationarity of local solutions of $(\mathrm{P})$.

This regularization concept then can be used to devise numerically implementable algorithms [16], that generate a sequence of solutions $\left\{\left(y_{c}, u_{c}\right)\right\}$. In [16] it was reported that the sequence of regularized controls converged in numerical experiments as

$$
\left\|u_{c}-u^{*}\right\|_{L^{2}(\Omega)}=O\left(c^{-1 / 2}\right) .
$$

We will prove this rate under assumptions on second-order information, see Theorem 4.5. This explains the numerical observations, and in turn the comparison with numerics shows that our rates of convergence are sharp. Such a quantitative insight is helpful in the construction of adaptive path-following algorithms. To the best of our knowledge, similar estimates are not known for related methods.

Moreover, we prove for the objective function the convergence rate

$$
\left|J\left(y_{*}, u_{*}\right)-J\left(y_{c}, u_{c}\right)\right|=O\left(c^{-1}\right),
$$

which results from an improved estimate for the derivative of the value function $V(c):=J\left(y_{c}, u_{c}\right)$. This estimate which can be found in Theorem 4.3, reads

$$
\left|\frac{\mathrm{d}}{\mathrm{d} c} V(c)\right|=O\left(c^{-2}\right) .
$$

This is a significant improvement, compared to the results in [16].

To summarize, our findings complement and extend the results obtained in [16]. Our most significant new contributions are $C$-stationarity of the limiting solutions, even in the presence of control constraints, and the sharp, quantitative estimate for the convergence rates of the regularization path in Theorem 4.5. 


\section{Standing assumptions}

Throughout the paper we rely on the following regularity assumptions.

(A.i) The domain $\Omega \subset \mathbb{R}^{n}, n \in\{2,3\}$ is a bounded Lipschitz domain.

(A.ii) The operator $A$ is an elliptic differential operator defined by

$$
(A y)(x)=-\sum_{i, j=1}^{n} \frac{\partial}{\partial x_{i}}\left(a_{i j}(x) \frac{\partial}{\partial x_{j}} y(x)\right)+\sum_{j=1}^{n} a_{j}(x) \frac{\partial}{\partial x_{j}} y(x)+a_{0}(x) y(x)
$$

with functions $a_{i j} \in C^{0,1}(\bar{\Omega}), a_{j}, \frac{\partial}{\partial x_{j}} a_{j}, a_{0} \in L^{\infty}(\Omega)$ satisfying the conditions $a_{i j}(x)=a_{j i}(x)$ and

$$
\sum_{i, j=1}^{n} a_{i j}(x) \xi_{i} \xi_{j} \geq \delta_{0}|\xi|^{2} \quad \text { a.e. on } \quad \Omega \quad \text { for all } \quad \xi \in \mathbb{R}^{n}
$$

with some $\delta_{0}>0$. Additionally, we require $a_{0}(x) \geq \delta_{1} \geq 0$ with $\delta_{1}$ sufficiently large such that $A$ fulfills the coercivity condition

$$
\nu_{1}\|v\|_{H_{0}^{1}}^{2} \leq\langle A v, v\rangle \quad \forall v \in H_{0}^{1}(\Omega) .
$$

(A.iii) The obstacle $\psi \in H^{1}(\Omega) \cap L^{\infty}(\Omega)$ fulfills $A \psi \in L^{\infty}(\Omega)$ and $\psi \geq 0$ on $\Gamma$.

The functions $g, j$ satisfy:

(A.iv) $g: L^{2}(\Omega) \rightarrow \mathbb{R}$ is twice continuously Fréchet-differentiable,

(A.v) $j: L^{2}(\Omega) \rightarrow \mathbb{R}$ is twice continuously Fréchet-differentiable and weakly lower semi-continuous.

The function $\bar{\lambda}$ appearing in the regularization satisfies

(A.vi) $\bar{\lambda} \in L^{\infty}(\Omega), \bar{\lambda} \geq 0$ a.e. on $\Omega$.

Let us introduce the adjoint operator $A^{*}$ to $A$ by

$$
\left(A^{*} p\right)(x)=-\sum_{j=1}^{n} \frac{\partial}{\partial x_{j}}\left(\sum_{i=1}^{n} a_{i j}(x) \frac{\partial}{\partial x_{i}} p(x)+a_{j}(x) p(x)\right)+a_{0}(x) p(x) .
$$

\section{Assumptions on the smooth approximation of max}

We assume that the function $\max _{c}$ admits the following properties:

(B.i) $\max _{c}:(c, x) \mapsto \max _{c}(x),(c, x) \in(0,+\infty) \times \mathbb{R}$, is twice continuously differentiable with respect to $(c, x)$, (B.ii) $\max _{c}(x)=\max (0, x)$ for all $x$ with $|x| \geq 1 / 2 c, \max _{c}(x) \geq \max (0, x)$ for all $x$ with $|x| \leq 1 / 2 c$.

We will denote the derivatives with respect to $x$ by $\max _{c}^{\prime}$, $\max _{c}^{\prime \prime}$, whereas the derivatives with respect to $c$ are denoted by $\frac{\partial}{\partial c} \max _{c}$.

In addition we assume that there is a constant $M>0$ such that the following inequalities are satisfied for all $x$ :

(B.iii) $0 \leq \max _{c}^{\prime}(x) \leq 1$,

(B.iv) $0 \leq \max _{c}^{\prime \prime}(x)$,

(B.v) $\left|\frac{\partial}{\partial c} \max _{c}(x)\right| \leq \frac{M}{c^{2}}$,

Note, that the function

$$
m_{c}(x):=\left\{\begin{array}{lll}
\max (0, x) & \text { if } & |x| \geq \frac{1}{2 c} \\
\frac{c^{3}}{2}\left(x+\frac{1}{2 c}\right)^{3}\left(\frac{3}{2 c}-x\right) & \text { if } & |x|<\frac{1}{2 c}
\end{array}\right.
$$


satisfies the requirements above, see [16]. Different kind of smooth approximations of $\max (0, \cdot)$ were used by Hintermüller and Kopacka [9].

A simple consequence of the monotonicity of $\max _{c}^{\prime}$ provided by (B.iv) is the following inequality

$$
\max _{c}(x) \leq\left(x+\frac{1}{2 c}\right) \max _{c}^{\prime}(x) \quad \forall x \in \mathbb{R} .
$$

\section{Notational convention}

We will use in several places generic constants, all denoted by $K$. These constants are independent of $c$ and sequences $\left\{\left(y_{c}, u_{c}\right)\right\}$ of local solutions of $\left(\mathrm{P}_{c}\right)$.

\section{Regularization of the obstacle problem}

Let us first recall the well-known existence and regularity results for the variational inequality (1.1), for the proofs we refer to $[4,15]$. Then we prove additional results for the regularized obstacle problem that we will need later.

Proposition 2.1. Let $u \in L^{2}(\Omega)$ be given. Then the variational inequality (1.1) admits a unique solution $y \in H_{0}^{1}(\Omega) \cap L^{\infty}(\Omega)$. The mapping $u \mapsto y$ is Lipschitz continuous. Moreover, it holds $\lambda, A y \in L^{2}(\Omega)$.

In addition, the mapping $u \mapsto y$ is directionally differentiable in a certain sense, see e.g. [18], but not Gâteaux differentiable.

\subsection{Uniform boundedness of solutions}

Let us now study the regularized equation

$$
A y+\max _{c}(\bar{\lambda}+c(y-\psi))=u,
$$

for fixed $c$ and given $u \in L^{2}(\Omega)$. Since the function $\max _{c}$ is monotone, we have the following existence and uniqueness result.

Proposition 2.2. Let $u \in L^{2}(\Omega)$ be given. Then (2.11) admits a unique solution $y_{c} \in H_{0}^{1}(\Omega) \cap L^{\infty}(\Omega)$. There is a constant $K>0$ independent of $c$ such that $\left\|y_{c}\right\|_{H_{0}^{1}}+\left\|y_{c}\right\|_{L^{\infty}} \leq K\|u\|_{L^{2}}$ for all $c>0$.

Proof. Existence and uniqueness of solutions follow from standard arguments. To obtain uniform bounds, one tests $(2.11)$ by $y_{c}$ and uses the uniform boundedness of $\max _{c}(\bar{\lambda}-c \psi)$.

We are interested in the asymptotic behavior of the solutions of (2.11) for $c \rightarrow \infty$. In the next lemma we will show that the constraint violation $\left(y_{c}-\psi\right)_{+}$tends to zero for $c \rightarrow \infty$. This improves earlier results of $[13,17]$. Here, $(v)_{+}$refers to the positive part of $v$, i.e. $(v)_{+}(x)=\max (0, v(x))$.

Lemma 2.3. Let $u \in L^{2}(\Omega)$ be given. Let $y_{c, u}$ denote the corresponding solution of the regularized equation (2.11).

If for some $2 \leq q \leq \infty$ it holds $u+\max (0,-A \psi-\bar{\lambda}) \in L^{q}(\Omega)$, then we have the estimates

$$
\begin{gathered}
\left\|\left(y_{c, u}-\psi\right)_{+}\right\|_{L^{q}} \leq c^{-1}\|u+\max (0,-A \psi-\bar{\lambda})\|_{L^{q}}, \\
\left\|\left(y_{c, u}-\psi\right)_{+}\right\|_{H_{0}^{1}} \leq K c^{-1 / 2}
\end{gathered}
$$

and

$$
\left\|A y_{c, u}\right\|_{L^{q}}+\left\|\max _{c}\left(\bar{\lambda}+c\left(y_{c, u}-\psi\right)\right)\right\|_{L^{q}} \leq K
$$


Proof. The proof follows to some extent the lines of the proof of [15], Lemma 5.1. Clearly, the function $\left(y_{c, u}-\psi\right)_{+}^{q}$ belongs to $H_{0}^{1}(\Omega)$ for all $q \geq 2$, which follows from the chain rule taking into account the boundedness of $y_{c, u}$ and $\psi$. Hence, we can test the equation

$$
A\left(y_{c, u}-\psi\right)+\max _{c}\left(\bar{\lambda}+c\left(y_{c, u}-\psi\right)\right)=u-A \psi,
$$

which is equivalent to $(2.11)$, by $\left(y_{c, u}-\psi\right)_{+}^{q-1}$.

By assumption (A.ii), we have for every $z \in L^{\infty} \cap H_{0}^{1}(\Omega)$

$$
\left\langle A z, z^{q-1}\right\rangle \geq \frac{2}{q}\left\langle A z^{q / 2}, z^{q / 2}\right\rangle+\left(1-\frac{2}{q}\right) \int_{\Omega} a_{0}|z|^{q} \geq \frac{2 \nu_{1}}{q}\left\|z^{q / 2}\right\|_{H^{1}}^{2}
$$

Using the fact that $\max _{c}(x) \geq \max (0, x) \geq x$ for all $x \in \mathbb{R}, c f$. (B.ii), we obtain

$$
\int_{\Omega} \max _{c}\left(\bar{\lambda}+c\left(y_{c, u}-\psi\right)\right)\left(y_{c, u}-\psi\right)_{+}^{q-1} \geq c\left\|\left(y_{c, u}-\psi\right)_{+}\right\|_{L^{q}}^{q}+\int_{\Omega} \bar{\lambda}\left(y_{c, u}-\psi\right)_{+}^{q-1} .
$$

Combining these results yields

$$
\begin{aligned}
c\left\|\left(y_{c, u}-\psi\right)_{+}\right\|_{L^{q}}^{q} & \leq\left(u-A \psi-\bar{\lambda},\left(y_{c, u}-\psi\right)_{+}^{q-1}\right) \\
& \leq\|u+\max (0,-A \psi-\bar{\lambda})\|_{L^{q}}\left\|\left(y_{c, u}-\psi\right)_{+}\right\|_{L^{q}}^{q-1},
\end{aligned}
$$

which gives

$$
c\left\|\left(y_{c, u}-\psi\right)_{+}\right\|_{L^{q}} \leq\|u+\max (0,-A \psi-\bar{\lambda})\|_{L^{q}} .
$$

This proves the first claim for $2 \leq q<\infty$. If $u+\max (0,-A \psi-\bar{\lambda}) \in L^{\infty}(\Omega)$ then we can pass to the limit $q \rightarrow \infty$ in (2.13), which gives the wanted $L^{\infty}(\Omega)$-estimate, and (2.12) is proven. The estimate for the $H^{1}$-norm is an easy consequence of the estimates for $q=2$.

Next, we write

$$
A y_{c, u}=u-\max _{c}\left(\bar{\lambda}+c\left(y_{c, u}-\psi\right)\right) .
$$

By (1.10) we have the estimate

$$
\max _{c}\left(\bar{\lambda}+c\left(y_{c, u}-\psi\right)\right)(x) \leq \frac{1}{2 c}+\bar{\lambda}(x)+c\left(y_{c, u}(x)-\psi(x)\right) .
$$

Hence it holds $\left\|\max _{c}\left(\bar{\lambda}+c\left(y_{c, u}-\psi\right)\right)\right\|_{L^{q}} \leq K$ by (2.12), with a constant $K>0$ independent of $q$ and $c>2$. This implies $\left\|A y_{c, u}\right\|_{L^{q}} \leq K$.

\subsection{The linearized equation}

Now let us consider the linearized version of (2.11)

$$
A w+c \max _{c}^{\prime}\left(\bar{\lambda}+c\left(y_{c}-\psi\right)\right) w=r_{c},
$$

where $y_{c}$ is a given solution of the regularized equation (2.11), and $r_{c} \in L^{2}(\Omega)$ is a given right-hand side. Regarding existence and boundedness of solutions we have the following:

Theorem 2.4. Let $\left\{y_{c}\right\}_{c>0}$ and $\left\{r_{c}\right\}_{c>0}$ be bounded in $H_{0}^{1}(\Omega)$ and $L^{2}(\Omega)$, respectively. Then equation (2.14) admits for each $c>0$ a unique solution $w_{c} \in H_{0}^{1}(\Omega) \cap L^{\infty}(\Omega)$, and there is a constant $K>0$ independent of $c$ such that

$$
\begin{aligned}
\left\|w_{c}\right\|_{H^{1}}+\left\|\sqrt{c \max _{c}^{\prime}} w_{c}\right\|_{L^{2}} & \leq K\left\|\left(1+c \max _{c}^{\prime}\right)^{-1 / 2} r_{c}\right\|_{L^{2}} \\
\left\|c \max _{c}^{\prime} w_{c}\right\|_{L^{1}} & \leq K\left\|r_{c}\right\|_{L^{1}} \\
\left\|w_{c}\right\|_{L^{\infty}} & \leq K\left\|r_{c}\right\|_{L^{2}} .
\end{aligned}
$$


Moreover, if $\left(\max _{c}^{\prime}\right)^{-1} r_{c} \in L^{\infty}(\Omega)$, then we obtain

$$
\left\|w_{c}\right\|_{L^{\infty}} \leq K\left\|\left(c \max _{c}^{\prime}\right)^{-1} r_{c}\right\|_{L^{\infty}}
$$

Here, we abbreviated $c \max _{c}^{\prime}:=c \max _{c}^{\prime}\left(\bar{\lambda}+c\left(y_{c}-\psi\right)\right)$.

Proof. For our first result, we test (2.14) by $w_{c}$, and divide by the square-root of the left-hand side. The second assertion follows by testing with a smoothed-sign function similarly as in [13], Theorem 5.1. The $L^{\infty}(\Omega)$-bound follows from the result of Stampacchia [22].

For our last result, we use a duality technique. For each $s \in L^{2}(\Omega)$ let $z_{s}=\left(A+c \max _{c}^{\prime}\right)^{-1} s$

$$
\begin{aligned}
\left\|w_{c}\right\|_{L^{\infty}} & =\sup _{s \in L^{2},\|s\|_{L^{1}} \leq 1}\left\langle w_{c}, s\right\rangle_{L^{2}} \\
& =\sup _{s}\left\langle\left(A+c \max _{c}^{\prime}\right)^{-1} r_{c}, s\right\rangle=\sup _{s}\left\langle r_{c}, z_{s}\right\rangle \\
& \leq \sup _{s}\left\|\left(c \max _{c}^{\prime}\right)^{-1} r_{c}\right\|_{L^{\infty}}\left\|c \max _{c}^{\prime} z_{s}\right\|_{L^{1}} \leq K\left\|\left(c \max _{c}^{\prime}\right)^{-1} r_{c}\right\|_{L^{\infty}},
\end{aligned}
$$

which finishes the proof.

We state a simple consequence of our previous result:

Corollary 2.5. The mapping $u \rightarrow y_{c, u}$ is Lipschitz continuous in the following sense:

$$
\left\|A\left(y_{c, u_{1}}-y_{c, u_{2}}\right)\right\|_{L^{1}}+\left\|y_{c, u_{1}}-y_{c, u_{2}}\right\|_{H^{1}}+\left\|y_{c, u_{1}}-y_{c, u_{2}}\right\|_{L^{\infty}} \leq L\left\|u_{1}-u_{2}\right\|_{L^{2}}
$$

with a Lipschitz constant $L$ that is independent of $c$. Moreover, we have the c-dependent Lipschitz estimate:

$$
\left\|A\left(y_{c, u_{1}}-y_{c, u_{2}}\right)\right\|_{L^{2}} \leq K \sqrt{c}\left\|u_{1}-u_{2}\right\|_{L^{2}}
$$

Proof. This follows from the implicit function theorem and the mean value theorem, applied to the path-following problem

$$
A y(t)+\max _{c}(\bar{\lambda}+c(y(t)-\psi))=t u_{2}+(1-t) u_{1},
$$

with $t \in[0,1]$. Estimates on the slope of this path are obtained via the linearized equation, using the estimates (2.15) and (2.16), which are independent of $c$. Hence, we conclude (2.17) with $L$ independent of $c$, as well. Our second estimate follows similarly via inserting the estimate

$$
\left\|c \max _{c}^{\prime}\left(\bar{\lambda}+c\left(y_{c}-\psi\right)\right) w\right\|_{L_{2}} \leq K c^{1 / 2}
$$

which is a consequence of (2.15), into (2.14).

Remark 2.6. Our estimates indicate that the mapping $u \rightarrow y_{c, u}$ cannot be expected to be Lipschitz-continuous from $L^{2}(\Omega)$ to $H^{2}(\Omega)$, uniformly in $c$. Still, by interpolation, we obtain the following uniform result on Hölder continuity:

$$
\left\|A\left(y_{c, u_{1}}-y_{c, u_{2}}\right)\right\|_{L^{2}} \leq K\left\|A\left(y_{c, u_{1}}-y_{c, u_{2}}\right)\right\|_{L^{\infty}}^{1 / 2}\left\|A\left(y_{c, u_{1}}-y_{c, u_{2}}\right)\right\|_{L^{1}}^{1 / 2} \leq K\left\|u_{1}-u_{2}\right\|_{L^{2}}^{1 / 2}
$$

provided $u_{i}+\max (0,-A \psi-\bar{\lambda}) \in L^{\infty}(\Omega)$ holds for $i=1,2$. 


\subsection{Convergence results for fixed $u$}

Let now $u \in L^{\infty}(\Omega)$ be fixed and $y_{c, u}$ the solution of the regularized obstacle problem (2.11) for parameter $c$. We consider the limiting behavior of $J\left(y_{c, u}, u\right)$ for $c \rightarrow \infty$. To this end, we differentiate the equation

$$
A y_{c, u}+\max _{c}\left(\bar{\lambda}+c\left(y_{c, u}-\psi\right)\right)=u
$$

with respect to $c$, and obtain

$$
\begin{aligned}
0= & A \dot{y}+c \max _{c}^{\prime}\left(\bar{\lambda}+c\left(y_{c, u}-\psi\right)\right) \dot{y} \\
& +\frac{\partial}{\partial c} \max _{c}\left(\bar{\lambda}+c\left(y_{c, u}-\psi\right)\right)+\max _{c}^{\prime}\left(\bar{\lambda}+c\left(y_{c, u}-\psi\right)\right)\left(y_{c, u}-\psi\right)=0 .
\end{aligned}
$$

Hence, the derivative $\dot{y}$ of $y_{c, u}$ with respect to $c$ is the solution of a linearized equation.

Let us define the following family of sets that will play an important role in the subsequent analysis

$$
\mathcal{P}_{c, u}=\left\{x \in \Omega: \bar{\lambda}(x)+c\left(y_{c, u}(x)-\psi(x)\right)>-\frac{1}{2 c}\right\} .
$$

That is, $\mathcal{P}_{c, u}$ contains the points, where $\max _{c}\left(\bar{\lambda}+c\left(y_{c, u}-\psi\right)\right)>0$. Obviously $\max _{c}\left(\bar{\lambda}+c\left(y_{c, u}-\psi\right)\right)=0$ on $\Omega \backslash \mathcal{P}_{c, u}$, and thus also $\max _{c}^{\prime}=0$ there.

Lemma 2.7. It holds

$$
\left\|y_{c, u}-\psi\right\|_{L^{\infty}\left(\mathcal{P}_{c, u}\right)} \leq K c^{-1}
$$

with a constant $K>0$ independent of $c$.

Proof. By definition of $\mathcal{P}_{c, u}$ it follows

$$
\frac{1}{c}\left(-\bar{\lambda}-\frac{1}{2 c}\right) \leq y_{c, u}-\psi \leq\left(y_{c, u}-\psi\right)_{+} \cdot
$$

Since $\left\|\left(y_{c, u}-\psi\right)_{+}\right\|_{L^{\infty}} \leq K c^{-1}$ by Lemma 2.3 and assumption (A.iii), the desired result follows.

Remark 2.8. Let us remark that the bound $\left\|y_{c, u}-\psi\right\|_{L^{\infty}\left(\mathcal{P}_{c, u}\right)}=O\left(c^{-1}\right)$ is sharp in the following sense. Suppose it holds $\left\|y_{c, u}-\psi\right\|_{L^{q}\left(\mathcal{P}_{c, u}\right)}=o\left(c^{-1}\right)$. This implies on one hand the convergence of $\lambda_{c, u}:=\max _{c}\left(\bar{\lambda}+c\left(y_{c}-\psi\right)\right) \rightarrow$ $\bar{\lambda}$ in $L^{q}$. On the other hand, $\lambda_{c, u}$ converges to $\lambda_{u}$ in $H^{-1}(\Omega)$, where $\lambda_{u}$ is the multiplier in the obstacle problem associated to $u$, see Theorem 2.10 below. Hence, $\bar{\lambda}=\lambda_{u}$ is necessary to get $\left\|y_{c, u}-\psi\right\|_{L^{q}\left(\mathcal{P}_{c, u}\right)}=o\left(c^{-1}\right)$, which is unlikely, as this requires that the solution of the obstacle problem is known.

Similarly, the estimate $\left\|\left(y_{c, u}-\psi\right)_{+}\right\|_{L^{q}}=O\left(c^{-1}\right)$ of Lemma 2.3 is sharp. The relation $\left\|\left(y_{c, u}-\psi\right)_{+}\right\|_{L^{q}}=o\left(c^{-1}\right)$ implies $0 \leq \lambda_{u} \leq \bar{\lambda}$ a.e. on $\Omega$. That is, if $\lambda_{u}>\bar{\lambda}$ on a set of positive measure, then the estimate (2.12) is sharp.

The estimate of Lemma 2.7 will turn out to be essential for our final convergence estimate Theorem 4.5, as it allows to prove convergence rates of norms of $\dot{y}_{c}$ as well as of the value function $V$, see below Theorem 4.3.

Proposition 2.9. Let $\dot{y}$ be the solution of (2.18). Then we have the estimates

$$
\begin{aligned}
\|\dot{y}\|_{L^{\infty}(\Omega)} & \leq K c^{-2}, \\
\|\dot{y}\|_{H_{0}^{1}(\Omega)} & \leq K c^{-3 / 2} .
\end{aligned}
$$

Proof. By Theorem 2.4 we get

$$
\|\dot{y}\|_{L^{\infty}} \leq K\left(\left\|\left(c \max _{c}^{\prime}\right)^{-1} \max _{c}^{\prime} \cdot\left(y_{c, u}-\psi\right)\right\|_{L^{\infty}}+\left\|\frac{\partial}{\partial c} \max _{c}\right\|_{L^{2}}\right),
$$


where we omitted the argument $\left(\bar{\lambda}+c\left(y_{c, u}-\psi\right)\right)$ in the derivatives of $\max _{c}$. By (B.v) the second addend of the right hand side is of order $c^{-2}$. Further, by definition of $\mathcal{P}_{c, u}$ and Lemma 2.7 we have

$$
\left\|\left(c \max _{c}^{\prime}\right)^{-1} \max _{c}^{\prime} \cdot\left(y_{c, u}-\psi\right)\right\|_{L^{\infty}(\Omega)}=\left\|c^{-1} \cdot\left(y_{c, u}-\psi\right)\right\|_{L^{\infty}\left(\mathcal{P}_{c, u}\right)} .
$$

Thus, we obtain

$$
\|\dot{y}\|_{L^{\infty}(\Omega)} \leq K c^{-2} .
$$

Similarly, Theorem 2.4 also yields

$$
\|\dot{y}\|_{H^{1}} \leq K\left(\left\|\left(c \max _{c}^{\prime}\right)^{-1 / 2} \max _{c}^{\prime} \cdot\left(y_{c, u}-\psi\right)\right\|_{L^{2}}+\left\|\frac{\partial}{\partial c} \max _{c}\right\|_{L^{2}}\right),
$$

which implies with analogous arguments as above

$$
\|\dot{y}\|_{H^{1}} \leq K c^{-3 / 2} .
$$

With the help of estimates on $\dot{y}$ we can now study the convergence of $y_{c, u}$ for $c \rightarrow \infty$ and fixed control $u$.

Theorem 2.10. Let $u \in L^{2}(\Omega)$ be given. Let $y_{u}$ and $y_{c, u}$ denote the corresponding solutions of the variational inequality (1.1) and the regularized equation (2.11), respectively. Let $\lambda_{u}$ be the corresponding multiplier in (1.1) and set $\lambda_{c, u}:=\max _{c}\left(\bar{\lambda}+c\left(y_{c, u}-\psi\right)\right)$.

Then there is a constant $K>0$ independent of $c$ such that

$$
\begin{aligned}
\left\|y_{u}-y_{c, u}\right\|_{L^{\infty}} & \leq K c^{-1} \\
\left\|y_{u}-y_{c, u}\right\|_{H^{1}}+\left\|\lambda_{u}-\lambda_{c, u}\right\|_{H^{-1}} & \leq K c^{-1 / 2} .
\end{aligned}
$$

Moreover, we have an estimate on the function values:

$$
\left|J\left(y_{u}, u\right)-J\left(y_{c, u}, u\right)\right| \leq K c^{-1} .
$$

Proof. Our convergence results on $y$ follow from integration of (2.20) and (2.21) with respect to $c$. The estimate on $\lambda$ follows from inserting the estimates on $y$ into the difference of (1.1) and (2.11).

As for (2.22) we compute by the chain rule:

$$
\frac{\mathrm{d}}{\mathrm{d} c} J\left(y_{c, u}, u\right)=g^{\prime}\left(y_{c, u}\right) \dot{y}
$$

Inserting (2.20) we obtain

$$
\left|\frac{\mathrm{d}}{\mathrm{d} c} J\left(y_{c, u}, u\right)\right| \leq\left\|g^{\prime}\left(y_{c, u}\right)\right\|_{L^{1}(\Omega)}\|\dot{y}\|_{L^{\infty}(\Omega)} \leq K c^{-2} .
$$

Integration of this inequality with respect to $c$ yields the assertion of this theorem.

\section{C-Stationarity of solutions of $(\mathrm{P})$}

Let now $\left(y^{*}, u^{*}\right)$ be a strict local optimal solution of the original problem $(\mathrm{P})$. Then owing to the following result there exist a sequence of solutions of the regularized problem $\left(\mathrm{P}_{c}\right)$.

Proposition 3.1. Let $\left(y^{*}, u^{*}\right)$ be a strict local optimal solution of the original problem $(\mathrm{P})$. Then there exists a sequence $\left\{y_{c}, u_{c}\right\}$ of local solutions of the regularized problem with $\left(y_{c}, u_{c}\right)$ with

$$
\begin{array}{ccc}
y_{c} \rightarrow y^{*} & \text { in } & H_{0}^{1}(\Omega), \\
y_{c} \rightarrow^{*} y^{*} & \text { in } & L^{\infty}(\Omega), \\
u_{c} \rightarrow^{*} u^{*} & \text { in } & L^{\infty}(\Omega) .
\end{array}
$$

Moreover, for the associated multiplier $\lambda_{c}$ we have $\lambda_{c} \rightarrow \lambda^{*}$ in $H^{-1}(\Omega)$. 
Proof. The proof follows the lines of a similar result [17]. Here, weak lower semi-continuity of $j$ is needed. The uniform boundedness of $\left\{u_{c}\right\}$ in $L^{\infty}(\Omega)$ is a result of the control constraints.

In particular, $u_{c} \rightarrow u^{*}$ in $L_{2}(\Omega)$, which can also be shown in the absence of control constraints and which is all we need in the following.

Corollary 3.2. There is a subsequence such that $y_{c} \rightarrow y^{*}$ in $C(\bar{\Omega})$.

Proof. By Theorem 2.10, we have $y_{c, u^{*}} \rightarrow y^{*}$ strongly in $C(\bar{\Omega})$ as $c \rightarrow \infty$. Due to compact embeddings we also have $u_{c} \rightarrow u^{*}$ in $W^{-1,4}(\Omega)$. Using the result of [22], this implies $\left\|y_{c, u^{*}}-y_{c}\right\|_{L^{\infty}(\Omega)} \rightarrow 0$. This proves the claim.

As observed in [17] the convergence of $u_{c}$ to $u^{*}$ is strong in $L^{2}(\Omega)$ if $j(u)=\frac{\alpha}{2}\|u\|^{2}$. Alternatively, strong convergence can be achieved by adding a penalty term $\left\|u^{*}-u_{c}\right\|^{2}$ to the functional, see [19] and the remarks at the end of this section.

Since the problem $\left(\mathrm{P}_{c}\right)$ is smooth, there exists an adjoint state $p_{c}$ such that the first-order necessary optimality system (1.8) is satisfied. Now, let us prove the uniform boundedness of the dual quantities in the regularized problem.

Lemma 3.3. There is a constant $K$, such that

$$
\left\|p_{c}\right\|_{H^{1}}+\left\|p_{c}\right\|_{L^{\infty}}+\left\|c \max _{c}^{\prime}\left(\bar{\lambda}+\left(y_{c}-\psi\right)\right) p_{c}\right\|_{L^{1}} \leq K .
$$

Proof. Since $\left\{y_{c}\right\}$ is bounded in $L^{2}(\Omega)$, we have boundedness of $\left\{g^{\prime}\left(y_{c}\right)\right\}$ in $L^{2}(\Omega)$ as well by assumption (A.iv), and we can conclude from Theorem 2.4 the asserted $L^{1}(\Omega)$ and $L^{\infty}(\Omega)$ bounds for $p_{c}$.

See also related results in $[13,17]$. Let us remark, that the previous lemma implies uniform boundedness of the sequence $\left\{\mu_{c}\right\}$ in $L^{1}(\Omega)$, and thus weak* convergence to an element of $C(\bar{\Omega})^{*}$, i.e., a measure.

Summarizing these results, we obtain the following.

Proposition 3.4. Let $\left(y^{*}, u^{*}\right)$ be a strict local optimal solution of the original problem $(\mathrm{P})$. Then there exists a subsequence of $\left\{\left(y_{c}, u_{c}, \lambda_{c}, p_{c}, \mu_{c}\right)\right\}$ of stationary points of $\left(\mathrm{P}_{c}\right)$, which satisfy $(1.8)$, such that

$$
\begin{array}{rll}
y_{c} \rightarrow y^{*} & \text { in } & H_{0}^{1}(\Omega) \cap C(\bar{\Omega}), \\
u_{c} \rightarrow^{*} u^{*} & \text { in } & L^{\infty}(\Omega), \\
\lambda_{c} \rightarrow \lambda^{*} & \text { in } & H^{-1}(\Omega), \\
\lambda_{c} \rightarrow^{*} \lambda^{*} & \text { in } & L^{\infty}(\Omega), \\
p_{c} \rightarrow^{*} p^{*} & \text { in } & H_{0}^{1}(\Omega) \cap L^{\infty}(\Omega), \\
p_{c} \rightarrow p^{*} & \text { in } & L^{q}(\Omega) \forall q<\infty, \\
\mu_{c} \rightarrow^{*} \mu^{*} & \text { in } & H^{-1}(\Omega) \cap C(\bar{\Omega})^{*} .
\end{array}
$$

It remains to prove that the limit point $\left(y^{*}, u^{*}, \lambda^{*}, p^{*}, \mu^{*}\right)$ is a C-stationary point for $(\mathrm{P})$.

Proposition 3.5. It holds

$$
\begin{gathered}
\left\|\mu_{c}\left(y_{c}-\psi\right)\right\|_{L^{1}}=O\left(c^{-1}\right), \\
\left\|\lambda_{c} p_{c}\right\|_{L^{1}}=O\left(c^{-1}\right) .
\end{gathered}
$$

Moreover, we have for the limit

$$
\begin{array}{lcr}
\mu^{*}\left(y^{*}-\psi\right)=0 \quad \text { in } & C(\bar{\Omega})^{*} . \\
\lambda^{*} p^{*}=0 \quad \text { a.e. in } & \Omega .
\end{array}
$$


Proof. For the first claim, we obtain using the result of Lemma 2.7

$$
\left\|\mu_{c}\left(y_{c}-\psi\right)\right\|_{L^{1}}=\int_{\Omega}\left|\mu_{c}\left(y_{c}-\psi\right)\right| \leq\left\|\mu_{c}\right\|_{L^{1}}\left\|y_{c}-\psi\right\|_{L^{\infty}\left(\mathcal{P}_{c, u_{c}}\right)} \leq K c^{-1} .
$$

To prove the second assertion, we use the inequality $\max _{c}(x) \leq\left(x+\frac{1}{2 c}\right) \max _{c}^{\prime}(x), c f$. (1.10). Hence by Lemma 3.3

$$
\begin{aligned}
\int_{\Omega}\left|\lambda_{c} p_{c}\right| & \leq \int_{\mathcal{P}_{c, u_{c}}}\left|\left(\frac{1}{2 c}+\bar{\lambda}+c\left(y_{c}-\psi\right)\right) \max _{c}^{\prime}\left(\bar{\lambda}+c\left(y_{c}-\psi\right)\right) p_{c}\right| \\
& \leq K c^{-1}\left\|c \max _{c}^{\prime}\left(\bar{\lambda}+c\left(y_{c}-\psi\right)\right) p_{c}\right\|_{L^{1}} \leq K c^{-1} .
\end{aligned}
$$

By Proposition 3.4, we have that $\mu_{c}\left(y_{c}-\psi\right) \rightarrow^{*} \mu^{*}\left(y^{*}-\psi\right)$ in $C(\bar{\Omega})^{*}$. Moreover, for smooth test functions $\phi$ we have $\left\langle\lambda_{c}, \phi p_{c}\right\rangle \rightarrow\left\langle\lambda^{*}, \phi p^{*}\right\rangle$, since $\lambda_{c} \rightarrow \lambda^{*}$ in $H^{-1}(\Omega)$ and $p_{c} \rightarrow p^{*}$ in $H_{0}^{1}(\Omega)$. Using $\lambda^{*}, p^{*} \in L^{\infty}(\Omega)$ we prove $\lambda^{*} p^{*}=0$ a.e. on $\Omega$.

Lemma 3.6. We have

$$
A^{*} p^{*}+\mu^{*}=g^{\prime}\left(y_{*}\right)
$$

and

$$
\left\langle\mu^{*}, \zeta^{2} p^{*}\right\rangle \geq \limsup _{c \rightarrow \infty}\left\langle\mu_{c}, \zeta^{2} p_{c}\right\rangle \geq 0
$$

for all functions $\zeta \in W^{1, \infty}(\bar{\Omega})$.

Proof. Our first assertion follows from the fact that $A p_{c}$ and $\mu_{c}$ converge weakly in $H^{-1}$. Testing

$$
A^{*}\left(p_{c}-p^{*}\right)+\left(\mu_{c}-\mu^{*}\right)=g^{\prime}\left(y_{c}\right)-g^{\prime}\left(y_{*}\right)
$$

with $\zeta^{2}\left(p_{c}-p^{*}\right)$ yields

$$
\left\langle A^{*}\left(p_{c}-p^{*}\right), \zeta^{2}\left(p_{c}-p^{*}\right)\right\rangle+\left\langle\mu_{c}-\mu^{*}, \zeta^{2}\left(p_{c}-p^{*}\right)\right\rangle=\left(g^{\prime}\left(y_{c}\right)-g^{\prime}\left(y_{*}\right)\right)\left(\zeta^{2}\left(p_{c}-p^{*}\right)\right) .
$$

Since $g^{\prime}\left(y_{c}\right) \rightarrow g^{\prime}\left(y^{*}\right)$ strongly the right-hand side tends to zero for $c \rightarrow \infty$. For the first addend we have due to the properties of $A^{*}$, see assumption (A.ii),

$$
\left\langle A^{*}\left(p_{c}-p^{*}\right), \zeta^{2}\left(p_{c}-p^{*}\right)\right\rangle=\left\langle A^{*} \zeta\left(p_{c}-p^{*}\right), \zeta\left(p_{c}-p^{*}\right)\right\rangle+\int_{\Omega} \pi_{c} \zeta\left(p_{c}-p^{*}\right)
$$

with $\pi_{c} \in L^{2}(\Omega)$ given by

$$
\pi_{c}=2 \sum_{i, j=1}^{n} a_{i j}(x) \frac{\partial\left(p_{c}-p^{*}\right)}{\partial x_{i}} \frac{\partial \zeta}{\partial x_{j}}+\left(p_{c}-p^{*}\right) \sum_{j=1}^{n} a_{j} \frac{\partial \zeta}{\partial x_{j}} .
$$

Since $p_{c} \rightarrow p^{*}$ in $H_{0}^{1}(\Omega)$ and $p_{c} \rightarrow p^{*}$ in $L^{2}(\Omega)$, we find

$$
\int_{\Omega} \pi_{c} \zeta\left(p_{c}-p^{*}\right) \rightarrow 0
$$

for $c \rightarrow \infty$. Hence we obtain

$$
\begin{aligned}
0 & \geq \limsup \left\langle\mu_{c}-\mu^{*}, \zeta^{2}\left(p_{c}-p^{*}\right)\right\rangle \\
& =\lim \sup \left(\left\langle\mu_{c}, \zeta^{2} p_{c}\right\rangle+\left\langle\mu^{*}, \zeta^{2} p^{*}\right\rangle-\left\langle\mu_{c}, \zeta^{2} p^{*}\right\rangle-\left\langle\mu^{*}, \zeta^{2} p_{c}\right\rangle\right) \\
& =\limsup \left(\left\langle\mu_{c}, \zeta^{2} p_{c}\right\rangle-\left\langle\mu^{*}, \zeta^{2} p^{*}\right\rangle\right),
\end{aligned}
$$

where we have used $\left\langle\mu_{c}, \zeta^{2} p^{*}\right\rangle \rightarrow\left\langle\mu^{*}, \zeta^{2} p^{*}\right\rangle$ and $\left\langle\mu^{*}, \zeta^{2} p_{c}\right\rangle \rightarrow\left\langle\mu^{*}, \zeta^{2} p^{*}\right\rangle$. This holds, because $\zeta^{2} p_{c} \rightarrow \zeta^{2} p^{*}$ in $H_{0}^{1}(\Omega)$ and $\mu_{c} \rightarrow^{*} \mu^{*}$ in $H^{-1}(\Omega)$. 
Corollary 3.7. The distribution, defined by $v \rightarrow\left\langle v p^{*}, \mu^{*}\right\rangle_{H_{0}^{1}, H^{-1}}$ has a representation as a positive measure.

Proof. By (3.23) the above distribution is well defined and positive. Then [12], Theorem 2.1.7, yields the desired result.

Proposition 3.8. Let $\zeta \in C(\bar{\Omega})$ with $\zeta=0$ on $\left\{y^{*}=\psi\right\}$. Then it holds

$$
\left\langle\mu^{*}, \zeta\right\rangle_{C(\bar{\Omega}) *, C(\bar{\Omega})}=0 .
$$

Proof. Let us define $I_{\sigma}:=\left\{y^{*}<\psi-\sigma\right\}$ for $\sigma \geq 0$.

Let $\sigma>0$ be given. By strong convergence $y_{c} \rightarrow y^{*}$ in $C(\bar{\Omega})$ it follows that $\max _{c}{ }^{\prime}\left(\bar{\lambda}+c\left(y_{c}-\psi\right)\right)=0$ on the set $\left\{y^{*} \leq \psi-\sigma\right\}=I_{\sigma}$ for all $c>C_{\sigma}, C_{\sigma}$ sufficiently large. This implies $\mu_{c}=0$ on $I_{\sigma}$ for $c>C_{\sigma}$.

Let us take a nonnegative function $\zeta \in C(\bar{\Omega})$ with $\zeta=0$ on $\left\{y^{*}=\psi\right\}=I_{0}$. Then the function

$$
\zeta_{\sigma}:=\max \left(\zeta-\|\zeta\|_{L^{\infty}\left(I_{0} \backslash I_{\sigma}\right)}, 0\right)
$$

is continuous with support contained in $I_{\sigma}$. Hence $\left\langle\mu_{c}, \zeta_{\sigma}\right\rangle_{C^{*}, C}=0$ for $c$ sufficiently large. Due to $\mu_{c} \rightarrow^{*} \mu^{*}$ in $C(\bar{\Omega})^{*}$ it holds $\left\langle\mu^{*}, \zeta_{\sigma}\right\rangle_{C^{*}, C}=0$ for all $\sigma>0$.

By construction we have $\left\|\zeta-\zeta_{\sigma}\right\|_{L^{\infty}} \leq\|\zeta\|_{L^{\infty}\left(I_{0} \backslash I_{\sigma}\right)}$. Now let us argue that $\lim _{\sigma \backslash 0}\|\zeta\|_{I_{0} \backslash I_{\sigma}}=0$. If this would not be true, then there would exist $\epsilon>0$ and sequences $\sigma_{n} \searrow 0, x_{\sigma_{n}} \in I_{0} \backslash I_{\sigma_{n}}$ with $\zeta\left(x_{\sigma_{n}}\right)>\epsilon$. Since $\bar{\Omega}$ is compact, the sequence $\left\{x_{\sigma_{n}}\right\}$ admits an accumulation point $\tilde{x}$. By definition of $I_{\sigma}$ it follows that $\tilde{x}$ belongs to $\left\{y^{*}=\psi\right\}$. Hence $\zeta(\tilde{x})=0$, which leads to the contradiction.

This implies $\zeta_{\sigma} \rightarrow \zeta$ in $C(\bar{\Omega})$, which allows to pass to the limit to show $\left\langle\mu^{*}, \zeta\right\rangle_{C^{*}, C}=0$.

Theorem 3.9. Let $\left(y^{*}, u^{*}\right)$ be a strict local optimal solution of the original problem $(\mathrm{P})$. Let $\left\{\left(y_{c}, u_{c}, \lambda_{c}, p_{c}, \mu_{c}\right)\right\}$ be the sequence given by Proposition 3.4, which converges to $\left(y^{*}, u^{*}, \lambda^{*}, p^{*}, \mu^{*}\right)$.

Assume that for a subsequence $j^{\prime}\left(u_{c}\right) \rightarrow j^{\prime}\left(u^{*}\right)$ in $L^{2}(\Omega)$ holds.

Then $\left(y^{*}, u^{*}, \lambda^{*}, p^{*}, \mu^{*}\right)$ is a C-stationary point of $(\mathrm{P})$ that is it satisfies (1.5).

Proof. The claim is a direct consequence of the previous results. The assumption on the convergence $j^{\prime}\left(u_{c}\right) \rightarrow$ $j^{\prime}\left(u^{*}\right)$ then allows to pass to the limit in $(1.8 \mathrm{c})$.

In order to prove C-stationarity of all local solutions of $(\mathrm{P})$, we have to drop the assumption on strong convergence of $j^{\prime}\left(u_{c}\right)$. This can be done by considering a penalized problem as follows

$$
\left\{\begin{array}{c}
\min J(y, u)+\left\|u-u^{*}\right\|_{L^{2}}^{2} \\
\text { over }(y, u) \in H_{0}^{1}(\Omega) \times U_{a d} \text { subject to } \\
A y+\max _{c}(0, \bar{\lambda}+c(y-\psi))=u
\end{array}\right.
$$

With the same arguments as in $[17,19]$, we obtain the existence of a sequence $\left\{\left(\tilde{y}_{c}, \tilde{u}_{c}\right)\right\}$ of solutions of $\left(\tilde{\mathrm{P}}_{c}\right)$ with $\left(\tilde{y}_{c}, \tilde{u}_{c}\right) \rightarrow\left(y^{*}, u^{*}\right)$ strongly in $\left(H_{0}^{1}(\Omega) \cap L^{\infty}(\Omega)\right) \times L^{2}(\Omega)$. Besides an obvious modification of the necessary condition given in (1.8), all the results of this section remain valid. And we obtain

Theorem 3.10. Let $\left(y^{*}, u^{*}\right)$ be a local optimal solution of the original problem $(\mathrm{P})$. Then $\left(y^{*}, u^{*}, \lambda^{*}, p^{*}, \mu^{*}\right)$ is a C-stationary point of $(\mathrm{P})$. 


\section{Convergence estimates of the Path}

In the following, we will prove convergence rates for $c \rightarrow \infty$. Here we will rely on the following assumption on the path:

Assumption 4.1. Let us impose the following assumptions:

(i) The pair $\left(y^{*}, u^{*}\right)$ is a local solution of $(\mathrm{P})$ that satisfies a quadratic growth condition

$$
\left\|u-u_{*}\right\|_{L^{2}(\Omega)}^{2} \leq \alpha\left(J\left(y_{*}, u_{*}\right)-J(y(u), u)\right)
$$

for all $u$ in a neighborhood of $u_{*}$.

(ii) There is a sequence $\left\{\left(y_{c}, u_{c}\right)\right\}_{c>0} \in H_{0}^{1}(\Omega) \times L^{2}(\Omega)$ of local solutions of $\left(\mathrm{P}_{c}\right)$ converging strongly to $\left(y^{*}, u^{*}\right)$.

(iii) The optimality system (1.8) for $\left(y_{c}, u_{c}\right)$ is strongly regular for all $c>C_{0}$ in the sense of [3] for some $C_{0}<\infty$.

Some comments are in order. The quadratic growth condition on $\left(y^{*}, u^{*}\right)$ is fulfilled if $\left(y^{*}, u^{*}\right)$ fulfills a secondorder sufficient optimality condition. Sufficient optimality conditions were investigated in [17] for the special case $U_{a d}=L^{2}(\Omega)$. They can be transferred to the control-constrained case using the ideas of [5]. Then $\left(y^{*}, u^{*}\right)$ satisfies a quadratic growth condition, if $\left(y^{*}, u^{*}\right)$ is strongly stationary and the second-order derivative of the Lagrangian is positive definite on a certain cone.

The existence of the path was discussed in [17], see also the discussion in Section 2. The strong regularity of (1.8) is connected to the solvability of the linearization of (1.8). If the linearized system is uniquely solvable, and the solutions depend continuously on the data, then (1.8) is strongly regular, see e.g. $[3,6]$.

Let us define the optimal value function as the function value of $J$ along the path,

$$
V(c):=J\left(y_{c}, u_{c}\right)
$$

Lemma 4.2. The value function $V$ is continuously differentiable from $\left(C_{0},+\infty\right)$ to $\mathbb{R}$. Moreover we have

$$
\begin{aligned}
\dot{V}(c) & =\left(\frac{\partial}{\partial c} \max _{c}\left(\bar{\lambda}+c\left(y_{c}-\psi\right)\right)+\max _{c}^{\prime}\left(\bar{\lambda}+c\left(y_{c}-\psi\right)\right)\left(y_{c}-\psi\right), p_{c}\right) \\
& =\left(\frac{\partial}{\partial c} \max _{c}\left(\bar{\lambda}+c\left(y_{c}-\psi\right)\right), p_{c}\right)+c^{-1}\left(\mu_{c}, y_{c}-\psi\right) .
\end{aligned}
$$

Proof. The differentiability follows from the strong regularity of (1.8), see [3] and also [16]. The expression for $\dot{V}$ follows from the definition of $\mu_{c}$.

Theorem 4.3. Under the assumption imposed in this section it holds

$$
\dot{V}(c)=O\left(c^{-2}\right) \text {. }
$$

Proof. We will estimate the two summands in the right hand side of (4.25). For the first, let us define the set

$$
\mathcal{N}_{c}=\left\{x:\left|\bar{\lambda}(x)+c\left(y_{c}(x)-\psi(x)\right)\right|<\frac{1}{2 c}\right\} .
$$

On $\Omega \backslash \mathcal{N}_{c}$ we have $\frac{\partial}{\partial c} \max _{c}\left(\bar{\lambda}+c\left(y_{c}-\psi\right)\right)=0$. On $\mathcal{N}_{c}$ it holds by (B.v)

$$
\left|\frac{\partial}{\partial c} \max _{c}\left(\bar{\lambda}+c\left(y_{c}-\psi\right)\right)\right| \leq K c^{-2} \text {. }
$$

Uniform boundedness of $p_{c}$ in $L^{q}(\Omega)$ yields an $O\left(c^{-2}\right)$ bound for the first summand. 
Consider the second summand. Due to Lemma 3.3, the functions $\mu_{c}$ are bounded in $L^{1}(\Omega)$. By Lemma 2.7, we have $\left\|y_{c}-\psi\right\|_{L^{\infty}\left(\mathcal{P}_{c, u_{c}}\right)} \leq K c^{-1}$, where $\mathcal{P}_{c, u_{c}} \supset \operatorname{supp} \mu_{c}$. Hence,

$$
\left|\left(\mu_{c}, y_{c}-\psi\right)\right| \leq c^{-1}\left\|\mu_{c}\right\|_{L^{1}(\Omega)}\left\|y_{c}-\psi\right\|_{L^{\infty}\left(\mathcal{P}_{c, u_{c}}\right)} \leq K c^{-2}
$$

with a constant $K$ independent of $c$.

Remark 4.4. The result of Theorem 4.3 improves the estimate $\dot{V}(c)=o(1 / c)$ of [16]. Moreover, it explains the numerically observed convergence rate of $\dot{V}(c)=O\left(c^{-2}\right)$ of [16].

Let us now state and prove the main result of this section.

Theorem 4.5. Under the assumption imposed in this section it holds

$$
\begin{aligned}
\left|J\left(y_{*}, u_{*}\right)-J\left(y_{c}, u_{c}\right)\right| & \leq K c^{-1}, \\
\left|J\left(y_{*}, u_{*}\right)-J\left(y\left(u_{c}\right), u_{c}\right)\right| & \leq K c^{-1}, \\
\left\|y_{*}-y_{c}\right\|_{H^{1}}+\left\|y_{*}-y_{c}\right\|_{L^{\infty}}+\left\|u_{*}-u_{c}\right\|_{L^{2}} & \leq K c^{-1 / 2} .
\end{aligned}
$$

Proof. Integration of the estimate of $\dot{V}(c)$ in Theorem 4.3 with respect to $c$ yields the first estimate. The second estimate follows from (2.22) via

$$
J\left(y_{*}, u_{*}\right)-J\left(y\left(u_{c}\right), u_{c}\right)=J\left(y_{*}, u_{*}\right)-J\left(y_{c}, u_{c}\right)+J\left(y_{c}, u_{c}\right)-J\left(y\left(u_{c}\right), u_{c}\right) .
$$

The third inequality for $u_{c}-u_{*}$ follows from the growth condition (4.24):

$$
\left\|u_{c}-u_{*}\right\|^{2} \leq \alpha\left(J\left(y_{*}, u_{*}\right)-J\left(y\left(u_{c}\right), u_{c}\right)\right) \leq K c^{-1} .
$$

Finally, we can apply the triangle inequality:

$$
\begin{aligned}
\left\|y_{c}-y_{*}\right\|_{L^{\infty}} & \leq\left\|y_{c}-y_{c, u_{*}}\right\|_{L^{\infty}}+\left\|y_{c, u_{*}}-y_{*}\right\|_{L^{\infty}} \leq L\left\|u-u_{*}\right\|_{L^{2}}+K c^{-1}, \\
\left\|y_{c}-y_{*}\right\|_{H^{1}} & \leq\left\|y_{c}-y_{c, u_{*}}\right\|_{H^{1}}+\left\|y_{c, u_{*}}-y_{*}\right\|_{H^{1}} \leq L\left\|u-u_{*}\right\|_{L^{2}}+K c^{-1 / 2} .
\end{aligned}
$$

Here we used Theorem 2.10 and Corollary 2.5.

Remark 4.6. The convergence rate $\left\|u_{*}-u_{c}\right\|_{L^{2}} \leq K c^{-1 / 2}$ provided by Theorem 4.5 coincides with the rates observed in the numerical computations of [16]. In this sense, the result of Theorem 4.5 is sharp. See also our numerical results in the next section.

\section{NumERICAL EXPERIMENTS}

Let us report on the numerical results for the solution of the following problem: Minimize

$$
J(y, u)=\frac{1}{2}\left\|y-y_{d}\right\|_{L^{2}}^{2}+\frac{\alpha}{2}\|u\|_{L^{2}}^{2}
$$

subject to the variational inequality

$$
(\nabla y, \nabla v-\nabla y) \geq(u, v-y) \quad \forall v \in K
$$

and the control constraints

$$
u \in U_{a d}
$$

with $K$ and $U_{a d}$ as in (1.3) and (1.2), respectively. As domain we choose $\Omega=(0,1)^{2}$. The data for our example are given by

$$
y_{d}(x)=5 x_{1}+x_{2}-1, \quad \psi(x)=4\left(x_{1}\left(x_{1}-1\right)+x_{2}\left(x_{2}-1\right)\right)+1.5
$$



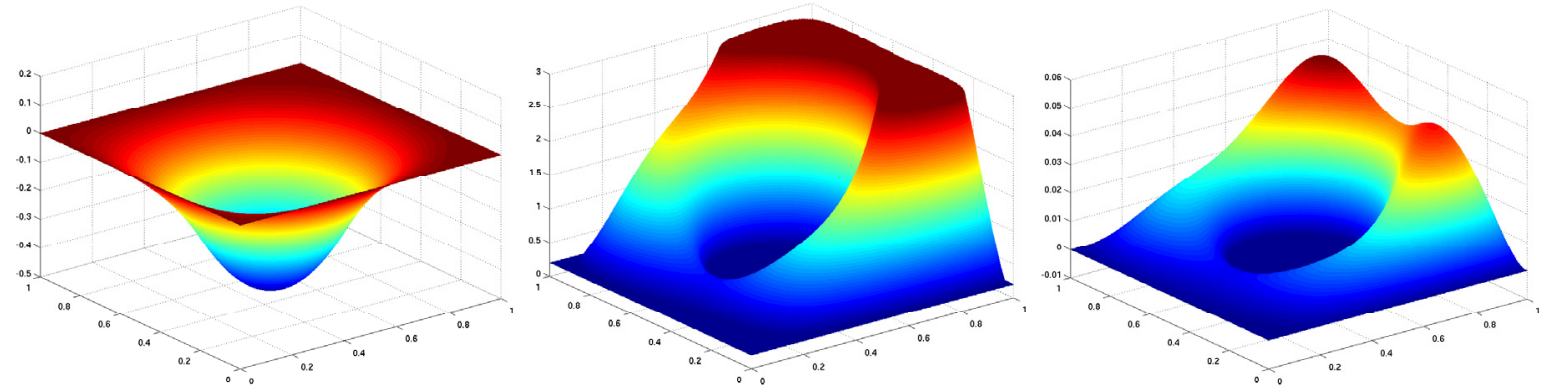

FiguRE 1. Numerical solution: $y_{c, h}, u_{c, h}, p_{c, h}$.
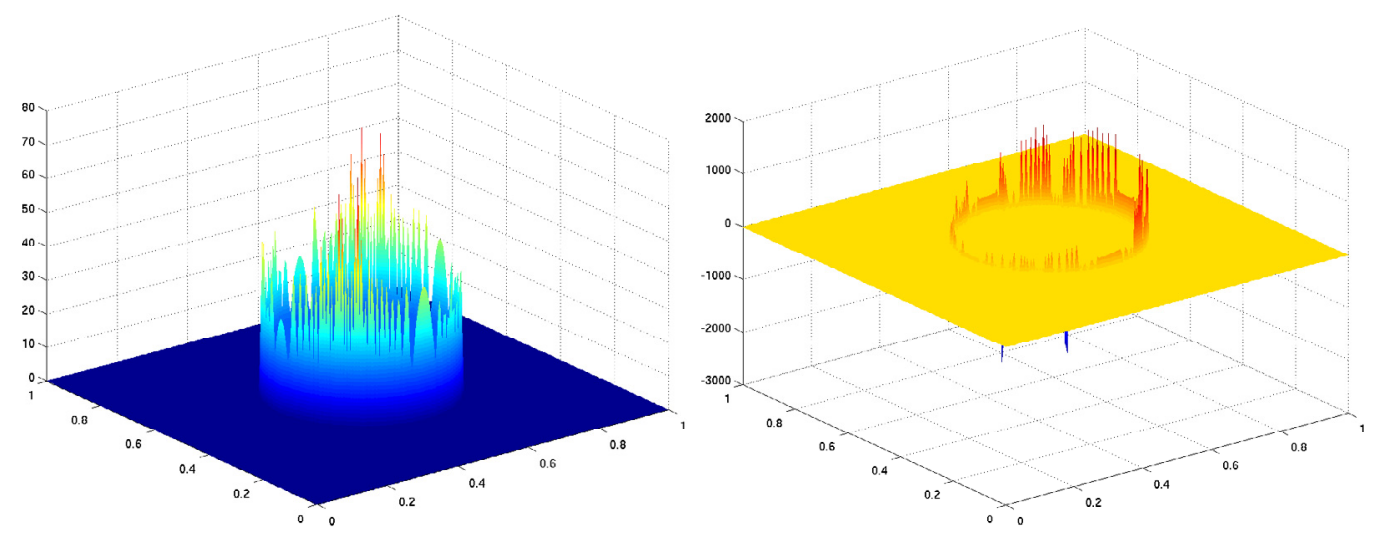

FiguRE 2. Numerical solution: $\lambda_{c, h}, \mu_{c, h}$.

and

$$
u_{a}=0.2, \quad u_{b}=3 .
$$

Furthermore, we set $\alpha=0.01$. With these choices, all the standing assumptions are satisfied.

We applied the inexact path-following strategy from [16] with tolerances $\epsilon_{\mathrm{cc}}=\epsilon_{\text {newt }}=10^{-8}$. We started the path-following algorithm with $c_{0}=100$, and set the parameter $\theta=0.5$.

The underlying partial differential equation was discretized by finite elements. We used $P 1$-elements for state, adjoint, and control discretization. The computational mesh consisted of 80000 triangles with maximal diameter $h=0.0071$.

The numerical solutions $\left(y_{c, h}, p_{c, h}, \lambda_{c, h}, \mu_{c, h}\right)$ for $c=c_{N} \approx 10^{9}$ are depicted in Figures 1 and 2. Both control and state constraints are active. As one can see, the adjoint state is zero on the active set. As shown in Figure 2, the multipliers $\lambda_{c, h}$ and $\mu_{c, h}$ only have low regularity. Moreover, the support of their irregular part is concentrated on the boundary of the active set $\{y=\psi\}$.

Let us now comment on the convergence rates for this example. The development of the convergence rates of $\left\|p_{c} \lambda_{c}\right\|_{L^{1}},\left\|\mu_{c}\left(y_{c}-\psi\right)\right\|_{L^{1}},\left\|u_{c}-u^{*}\right\|_{L^{2}}$, and $|\dot{V}(c)|$ are depicted in Figure 3 . As the solution of the continuous problem $u^{*}$ is unknown, we took the solution of the regularized problem for the largest value of $c_{N} \approx 10^{9}$ as reference solution, i.e. $u^{*}:=u_{c_{N}}$.

We observe the same convergence rates as predicted by the theory in Proposition 3.5, Theorem 4.3, and Theorem 4.5. 

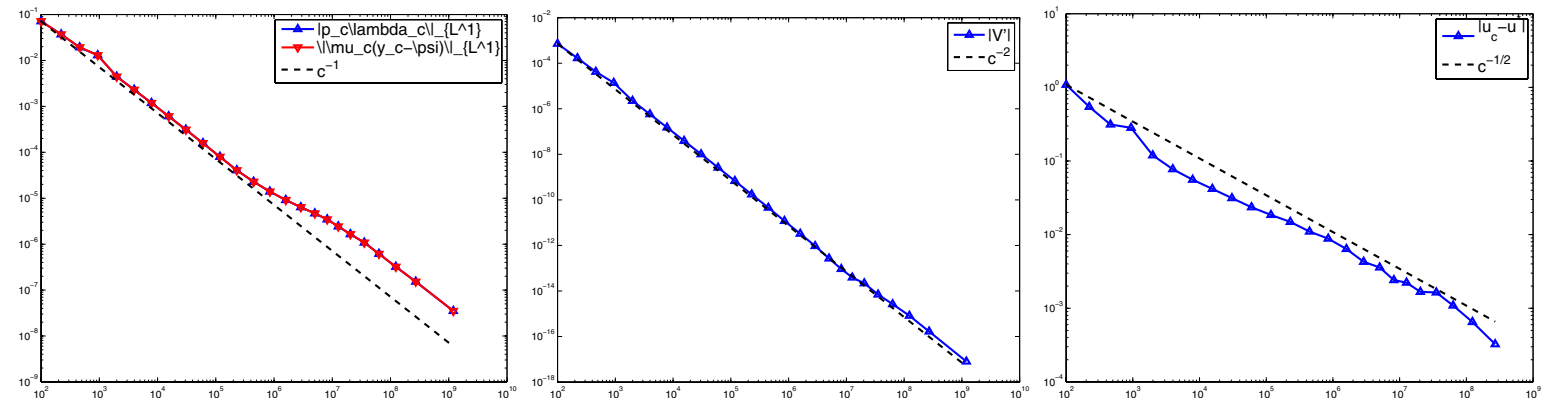

FiguRE 3. Convergence rates for $\left\|p_{c} \lambda_{c}\right\|_{L^{1}},\left\|\mu_{c}\left(y_{c}-\psi\right)\right\|_{L^{1}},|\dot{V}(c)|$, and $\left\|u_{c}-u^{*}\right\|_{L^{2}}$.

\section{REFERENCES}

[1] V. Barbu, Optimal control of variational inequalities, Monographs and Studies in Mathematics. Pitman (Advanced Publishing Program), London 24 (1984).

[2] M. Bergounioux and F. Mignot, Optimal control of obstacle problems: existence of Lagrange multipliers. ESAIM: COCV 5 (2000) 45-70.

[3] J.F. Bonnans and A. Shapiro, Perturbation analysis of optimization problems. Springer, New York (2000).

[4] H. Brezis and G. Stampacchia, Sur la régularité de la solution d'inéquations elliptiques. Bull. Soc. Math. France 96 (1968) 153-180.

[5] E. Casas, F. Tröltzsch and A. Unger, Second-order sufficient optimality conditions for a nonlinear elliptic control problem. J. Anal. Appl. 15 (1996) 687-707.

[6] A.L. Dontchev, Implicit function theorems for generalized equations. Math. Program. A70 (1995) 91-106.

[7] A. Friedman, Variational principles and free-boundary problems. Pure and Applied Mathematics. John Wiley \& Sons Inc., New York (1982).

[8] M. Hintermüller and I. Kopacka, Mathematical programs with complementarity constraints in function space: $C$ - and strong stationarity and a path-following algorithm. SIAM J. Optim. 20 (2009) 868-902.

[9] M. Hintermüller and I. Kopacka, A smooth penalty approach and a nonlinear multigrid algorithm for elliptic MPECs. Comput. Optim. Appl. 50 (2011) 111-145.

[10] M. Hintermüller, B.S. Mordukhovich and T. Surowiec, Several approaches for the derivation of stationarity conditions for elliptic MPECs with upper-level control constraints. IFB-Report No. 46 (07/2011), Institute of Mathematics and Scientific Computing, University of Graz.

[11] M. Hintermüller and T. Surowiec, First-order optimality conditions for elliptic mathematical programs with equilibrium constraints via variational analysis. SIAM J. Optim. 21 (2011) 1561-1593.

[12] L. Hörmander, The Analysis of Partial Differential Operators. Springer (1983).

[13] K. Ito and K. Kunisch, Optimal control of elliptic variational inequalities. Appl. Math. Optim. 41 (2000) 343-364.

[14] K. Ito and K. Kunisch, On the Lagrange multiplier approach to variational problems and applications, Monographs and Studies in Mathematics. SIAM, Philadelphia 24 (2008).

[15] D. Kinderlehrer and G. Stampacchia, An Introduction to Variational Inequalities and Their Applications. Academic Press, New York (1980).

[16] K. Kunisch and D. Wachsmuth, Path-following for optimal control of stationary variational inequalities. Comp. Opt. Appl. 51 (2011) 1345-1373.

[17] K. Kunisch and D. Wachsmuth, Sufficient optimality conditions and semi-smooth Newton methods for optimal control of stationary variational inequalities. ESAIM: COCV $\mathbf{1 8}$ (2012).

[18] F. Mignot, Contrôle dans les inéquations variationelles elliptiques. J. Funct. Anal. 22 (1976) $130-185$.

[19] F. Mignot and J.-P. Puel, Optimal control in some variational inequalities. SIAM J. Control Optim. 22 (1984) $466-476$.

[20] J. Outrata, J. Jarušek and J. Stará, On optimality conditions in control of elliptic variational inequalities. Set-Valued Var. Anal. 19 (2011) 23-42.

[21] H. Scheel and S. Scholtes, Mathematical programs with complementarity constraints: stationarity, optimality, and sensitivity. Math. Oper. Res. 25 (2000) 1-22.

[22] G. Stampacchia, Le problème de Dirichlet pour les équations elliptiques du second ordre à coefficients discontinus. Ann. Inst. Fourier (Grenoble) 15 (1965) 189-258.

[23] G. Wachsmuth, Private communication (2012). 